\title{
Aspectos motivacionais do abandono do tratamento de obesidade
}

\author{
Motivational aspects of dropout obesity treatment
}

\author{
Catia Suely Palmeira', Isadora Novais ${ }^{2}$, Victoria Roberta Silva Santos ${ }^{3}$, Maria de Lourdes Lima ${ }^{4}$ \\ ${ }^{1}$ Autora para correspondência. Escola Bahiana de Medicina e Saúde Pública. Salvador, Bahia, Brasil. \\ ORCID: 0000-0001-6328-8118. cspalmeira@bahiana.edu.br \\ 2Escola Bahiana de Medicina e Saúde Pública. Salvador, Bahia, Brasil. ORCID: 0000-0003-2527-3438. isa_6161@hotmail.com \\ ${ }^{3}$ Escola Bahiana de Medicina e Saúde Pública. Salvador, Bahia, Brasil. ORCID: 0000-0002-0243-3171. victoriaenfa@hotmail.com \\ ${ }^{4}$ Escola Bahiana de Medicina e Saúde Pública. Salvador, Bahia, Brasil. ORCID: 0000-0002-2081-4162. mlourdeslima@gmail.com
}

\begin{abstract}
RESUMO | OBJETIVO: Identificar os motivos que levaram ao abandono do tratamento de obesidade. MÉTODOS: Pesquisa descritiva com abordagem quantitativa realizada em um serviço de referência no acompanhamento de pessoas com excesso de peso na cidade de Salvador-BA. A população foi constituída por 62 pessoas que deixaram de frequentar o programa multidisciplinar por mais de dois anos. A coleta dos dados foi realizada pela análise do prontuário e entrevista por telefone. RESULTADOS: Predomínio do sexo feminino (90,3\%), com média de idade de 47,9 anos, cor parda $(46,8 \%)$, renda menor do que 3 salários mínimos $(64,7 \%)$ e escolaridade até o segundo grau (75,8\%). Depois da saída do programa $87,1 \%$ não estava sendo acompanhada por nenhum outro serviço de saúde para o tratamento do excesso de peso. Os motivos mais frequentes para o abandono foram de ordem pessoal como à falta de tempo (24,2\%) e problemas inerentes ao trabalho $(38,7 \%)$; e relacionados ao serviço, tais como o horário de atendimento $(24,2 \%)$, dificuldade em agendar retorno $(24,2 \%)$ e demora no atendimento (16,1\%). CONCLUSÃO: O abandono do tratamento da obesidade é complexo e causado por múltiplos fatores, relacionados a motivos de ordem pessoal, à estrutura e organização do serviço de saúde a equipe de profissionais.
\end{abstract}

PALAVRAS-CHAVE: Obesidade. Sobrepeso. Tratamento. Pacientes desistentes do tratamento.

\begin{abstract}
OBJECTIVES: To identify the reasons that led to quitting obesity treatment. METHOD: Descriptive research with quantitative approach conducted in a reference service at monitoring overweight people in the city of Salvador-BA. The population consisted of 62 people who stopped attending for over two years the multidisciplinary program. Data collection was developed by analyzing the medical records and interviewing by telephone. RESULTS: Predominance of women (90.3\%), with an average age of 47.9 years, brown skinned (46.8\%), income lower than 3 minimum wages (64.7\%) and education degree only up to high school (75.8\%). After leaving the program $87.1 \%$ of them were not being assisted by any other health service for overweight treatment. The most commum reasons for quitting were personal - such as lack of time (24.2\%) and problems inherent to work (38.7\%) - and related to the service - such as timetables (24.2\%), trobles to schedule feedbacks (24.2\%) and delays on the service (16.1\%). CONCLUSION: The abandonment of obesity treatment is complex and caused by multiple factors, related to personal reasons, structure and organization of professional team and healthcare services
\end{abstract}

KEYWORDS: Obesity. Overweight. Tratamento. Patient dropouts. 


\section{Introdução}

O excesso de peso é um problema de saúde pública que vem crescendo a cada dia na sociedade moderna, sendo considerado como uma pandemia, que atinge tanto país desenvolvido como em desenvolvimento, entre eles o Brasi ${ }^{1,2}$. De acordo com a pesquisa "Vigilância de fatores de risco e proteção para doenças crônicas por inquérito telefônico" (VIGITEL)³, em 2017 o número de pessoas com excesso de peso no Brasil atingiu $54,0 \%$ da população adulta, sendo que destes $18,9 \%$ tem obesidade.

O aumento de peso nas populações é reflexo dos processos históricos, sociais e culturais que as sociedades modernas vêm passando. Embora a obesidade esteja associada a questões genéticas, o seu desenvolvimento é fortemente influenciado por outros fatores ligados ao estilo de vida ${ }^{4}$. A mudança comportamental da população no que se refere ao consumismo, no qual as refeições rápidas e fora de casa substituíram os alimentos frescos e mais saudáveis, e a necessidade de prazeres rápidos contribuem para o aparecimento da obesidade como uma questão social|4,5.

A obesidade é uma doença crônica, caracterizada pelo excesso de gordura corporal causado pelo desequilíbrio entre consumo e gasto energético ${ }^{2}$. Tem múltiplas causas e decorre da interação entre fatores genéticos, ambientais e psicossociais ${ }^{4}$. Representa um agravo de saúde de alta complexidade, afeta todas as faixas etárias e grupos socioeconômicos com sérias repercussões clinicas e psicossociais. Relaciona-se diretamente com condições crônicas, como doenças cardiovasculares, hipertensão arterial sistêmica, diabetes mellitus tipo 2, apneia do sono, doença hepática gordurosa não alcoólica e vários tipos de câncer, sendo assim causa de incapacidade funcional, de redução da qualidade e da expectativa de vida, e aumento da mortalidade ${ }^{2}$.

Considera-se, portanto que as implicações do excesso de peso são vastas e vão além dos danos físicos, repercutindo principalmente nas dimensões psicológicas e sociais, pois se associa à discriminação, preconceitos e sentimentos de inferioridade, inadequação e depreciação, oportunidade de emprego, levando muitas vezes o indivíduo ao isolamento ${ }^{6}$.
O tratamento clínico não medicamentoso do excesso de peso é a primeira opção e visa mudanças no estilo de vida, abrangendo a prática de atividade física formal, aumento da atividade física informal e mudanças de hábito alimentar². A farmacoterapia deve servir apenas como um suporte no tratamento dietético e não como estrutura fundamental ${ }^{2}$, e o tratamento cirúrgico como um meio consistente nos casos de obesidade grave com falha de tratamento clínico ${ }^{7}$.

Devido à obesidade ser uma condição de difícil manejo, consequentemente, o seu tratamento será complexo, multiprofissional e interdisciplinar, pois, embora pareça simples, a redução de peso exige tratamentos de longa duração e um grande empenho do paciente ${ }^{8}$. Geralmente as altas taxas de insucesso do controle do peso estão associadas à baixa adesão aos programas terapêuticos ${ }^{2,9}$. Grande percentual de pessoas que recebem orientações de profissionais de saúde quanto às mudanças no estilo de vida, incluindo restrições alimentares, apresentam baixa adesão levando a maioria a fracassar no controle do peso ${ }^{9,10}$.

A adesão ao tratamento envolve uma complexidade de vários fatores que podem estar associados às características do usuário, a doença, aos hábitos culturais e de vida, ao tratamento, as dificuldades institucionais e a equipe de saúde ${ }^{9,11}$. Geralmente, tratamento em longo prazo resulta em baixa resposta ao acompanhamento. Em regra, o indivíduo obeso que procura tratamento tem um histórico de tentativas de perda de peso que na maioria das vezes tornam-se fracassadas, diante das mudanças no estilo de vida a que estão sujeitos ${ }^{9,12}$.

Nesse contexto a necessidade de conhecer quais os fatores motivacionais que levam os indivíduos a abandonarem o tratamento da obesidade é relevante, considerando principalmente a escassez de estudos sobre a temática. O conhecimento destes fatores pode colaborar para a prestação de uma assistência individualizada e qualificada, que por sua vez contribuirá para reduzir o abandono do tratamento.

Assim, este estudo teve como questão norteadora: Quais são os motivos que levaram ao abandono do tratamento da obesidade? E como objetivo identificar os motivos que levaram ao abandono do tratamento de obesidade. 


\section{Métodos}

Trata-se de uma pesquisa descritiva exploratória com abordagem quantitativa. O estudo foi realizado Centro Médico da Bahiana, localizado na cidade de Salvador, Bahia, Brasil, onde é desenvolvido o "Projeto de Excesso de Peso e Doenças Cardiometabólicas - PEPE" desde 2009. Este projeto acompanha pessoas com excesso de peso e suas comorbidades por meio de uma equipe de saúde constituída por médica, enfermeira, nutricionista, psicóloga e discentes de graduação e pós-graduação da área de saúde.

No período da coleta o PEPE tinha cadastrado 420 pacientes e sem frequentar o programa há mais de dois anos 186 indivíduos. A população do estudo foi constituída pelos pacientes desistentes do tratamento de obesidade no PEPE que atenderam os critérios de inclusão: há mais de dois anos sem frequentar o projeto, residentes da cidade de Salvador e regiões metropolitanas, e maiores de 18 anos. Foram excluídos os indivíduos que não dispõe de telefone, que não atenderam ao telefone após três tentativas, os incapazes de responder ao instrumento de coleta de dados, os falecidos e os que se recusaram. A amostra final resultou em 62 participantes (Figura 1).

Figura 1. Fluxograma de seleção dos participantes

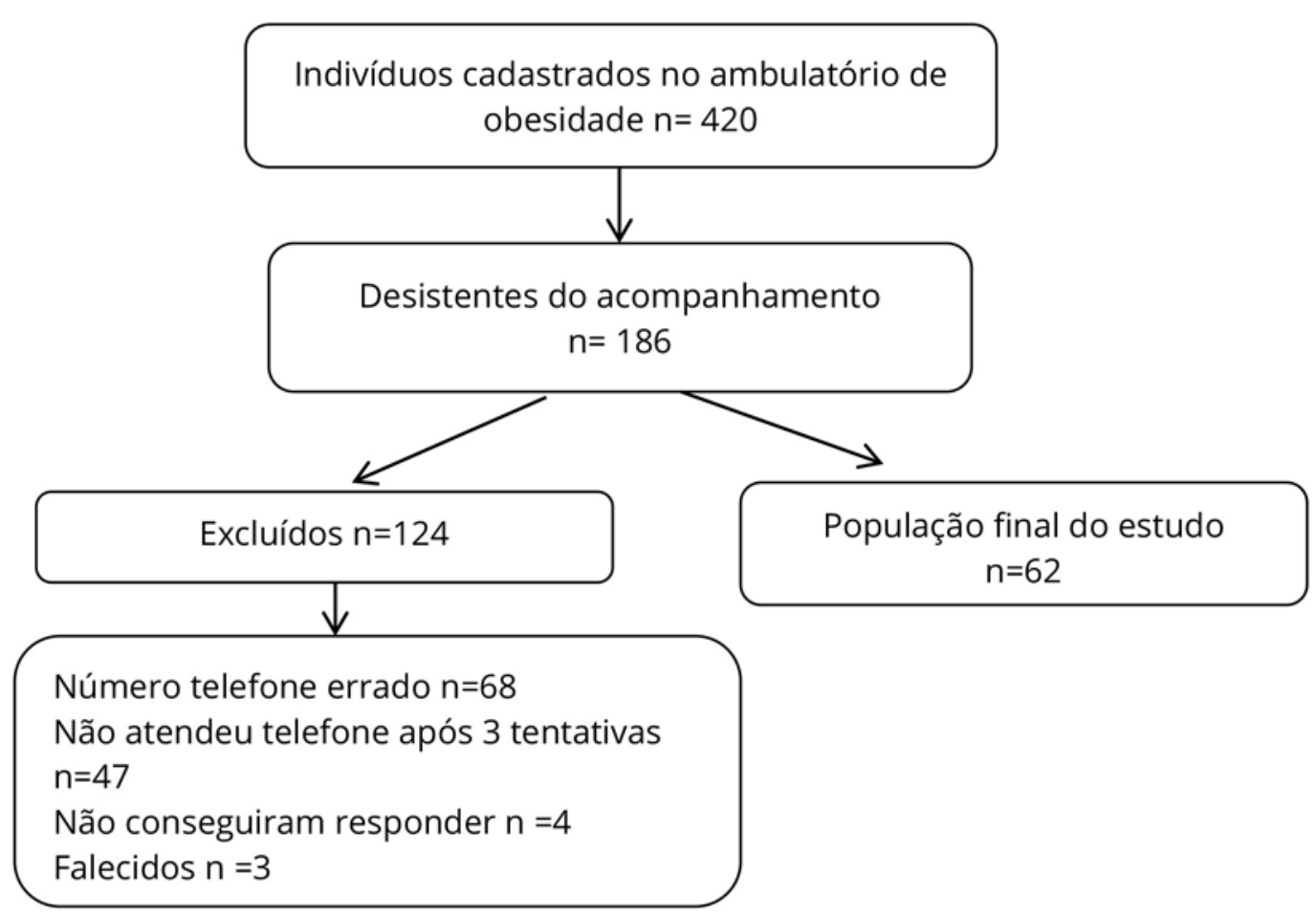

Os dados foram coletados no período de março de 2016 a setembro de 2016, por meio de um questionário estruturado elaborado pelas autoras com base em estudos sobre adesão ao tratamento, considerando que não existe um instrumento validado sobre o objeto de estudo. O instrumento foi constituído de duas partes: a parte I que incluiu os dados sociodemográficos, antropométricos e clínicos coletados no prontuário, e a parte Il que corresponde aos motivos do abandono, foi realizada por meio de entrevista por contato telefônico. 
Para caracterização da população foram utilizadas variáveis sociodemográficas já registradas no prontuário como: idade, sexo, ocupação, escolaridade, situação conjugal, renda e cor da pele; e as variáveis clínicas: comorbidades, tratamentos prévios, história do excesso de peso, peso, altura, IMC.

O IMC foi classificado de acordo com a relação entre a massa corporal e altura (kg/m2), seguindo os critérios da Organização Mundial de Saúde (OMS)13 para classificação de peso do indivíduo adulto: baixo peso $(<18,5)$ peso normal (<18,5 - 24,9); sobrepeso ( $\geq 25)$; sobrepeso (25 a 29,9) obeso I $(30$ a 34,9); obeso II (35 a 39,9); obeso III ( $\geq 40)$.

As questões sobre os motivos do abandono foram elaboradas com base na literatura sobre o objeto do estudo e para melhor compreensão da abrangência foram classificados nas seguintes categorias: relacionados ao serviço, relacionados a equipe de profissionais e relacionados a pessoa.

Os dados foram submetidos à análise estatística descritiva. As variáveis categóricas foram por meio de frequências absolutas percentuais e as quantitativas por meio de medidas de tendência central (média) e variabilidade (desvio-padrão) a depender da distribuição. Para armazenamento e processamento dos dados foi utilizado o programa Statistical Package for Social Science (IBM SPSS versão 18.0).

O projeto foi aprovado pelo Comitê de Ética em Pesquisa da Escola Bahiana de Medicina e Saúde Pública em 2009, ano da sua implantação, sob protocolo no 72/2009. O estudo respeitou as exigências formais contidas nas normas nacionais e internacionais regulamentadoras de pesquisas envolvendo seres humanos.

\section{Resultados}

Analisando as variáveis da caracterização sociodemográfica da população de estudo, os 62 participantes apresentam média de idade de 47,9 ( $D P=12,0)$, mínima de 22 e máxima de 79 anos, predominância a faixa etária de 40 a 59 anos, sexo feminino $(90,3 \%), 2^{\circ}$ grau de escolaridade (50\%), cor parda $(46,8 \%)$, situação conjugal com companheiro $(59,7 \%)$ e com renda media de 2,8 salários mínimos, variando de 0,5 a 16 salários mínimos (Tabela 1).

Tabela 1. Caracterização sociodemográfica de pessoas acompanhadas no Ambulatório de Obesidade, PEPE, na cidade de Salvador-Ba, 2019

\begin{tabular}{|c|c|c|}
\hline Variáveis & $\mathbf{n}$ & $\%$ \\
\hline \multicolumn{3}{|l|}{ Idade } \\
\hline $20-30$ & 19 & 30,6 \\
\hline $40-59$ & 33 & 53,2 \\
\hline$\geq 60$ & 10 & 16,1 \\
\hline \multicolumn{3}{|l|}{ Sexo } \\
\hline Masculino & 6 & 9,7 \\
\hline Feminino & 56 & 90,3 \\
\hline \multicolumn{3}{|l|}{ Escolaridade $(n=61)$} \\
\hline 1 grau & 14 & 22,6 \\
\hline 2 grau & 31 & 50,0 \\
\hline 3 grau & 16 & 25,8 \\
\hline \multicolumn{3}{|l|}{ Cor da pele $(n=60)$} \\
\hline Branco & 8 & 12,9 \\
\hline Pardo & 29 & 46,8 \\
\hline Negro & 23 & 37,1 \\
\hline \multicolumn{3}{|l|}{ Situação conjugal ( $n=62$ ) } \\
\hline Com companheiro & 37 & 59,7 \\
\hline Sem companheiro & 25 & 40,3 \\
\hline Renda em salários mínimos* (n-51) & 0,5 & 16,0 \\
\hline$<3 \mathrm{SM}$ & 33 & 64,7 \\
\hline$\geq 3 \mathrm{SM}$ & 18 & 35,3 \\
\hline
\end{tabular}

* Salário mínimo do ano de entrada no PEPE 
A média do IMC foi de $36,6 \mathrm{~kg} / \mathrm{m}^{2}$ ( $\mathrm{DP}=5,0$ ) com valor mínimo $25,5 \mathrm{~kg} / \mathrm{m} 2$ e máximo $46,9 \mathrm{~kg} / \mathrm{m} 2$. A obesidade grau II apresentou maior frequência (42,4\%). Entre os participantes 50,0\% tinham o diagnóstico de hipertensão arterial, 35,5\% de dislipidemia e 16,1\% de diabetes mellitus (Tabela 2). Quanto ao tempo de permanência no PEPE, observou-se que a maioria dos indivíduos (54,8\%) permaneceu no programa menos de um ano e $64,5 \%$ realizou de 1-4 consultas (Tabela 2 ).

Tabela 2. Caracterização de acordo com o IMC, comorbidades, tempo de permanência e número de consulta de pessoas acompanhadas no Ambulatório de Obesidade, PEPE, na cidade de Salvador-Ba, 2019

\begin{tabular}{|c|c|c|c|c|}
\hline Variável & Média & (*DP) & $\mathbf{n}$ & $\%$ \\
\hline IMC & 36,6 & 5,0 & & \\
\hline Sobrepeso & & & 6 & 10,2 \\
\hline Obesidade I & & & 16 & 27,1 \\
\hline Obesidade grau II & & & 25 & 42,4 \\
\hline Obesidade grau III & & & 12 & 20,3 \\
\hline \multicolumn{5}{|l|}{ Comorbidades } \\
\hline Hipertensão arterial & & & 31 & 50,0 \\
\hline Dislipidemia & & & 22 & 35,5 \\
\hline Diabetes & & & 10 & 16,1 \\
\hline DAC & & & 7 & 11,3 \\
\hline \multicolumn{5}{|l|}{ Tempo de PEPE } \\
\hline Menos de 1 ano & & & 34 & 54,8 \\
\hline $1-2$ anos & & & 21 & 33,9 \\
\hline 3-4 anos & & & 6 & 9,7 \\
\hline$\geq 5$ & & & 1 & 1,6 \\
\hline \multicolumn{5}{|l|}{ Número de consultas } \\
\hline $1-4$ & & & 40 & 64,5 \\
\hline $5-8$ & & & 12 & 19,4 \\
\hline$\geq 9$ & & & 10 & 16,1 \\
\hline
\end{tabular}

Os dados relacionados aos motivos para o não comparecimento ao PEPE encontram-se na Tabela 3. Com relação à organização e estrutura do serviço, as dificuldades em agendar o retorno e o horário do atendimento foram ambos citados por $24,2 \%$, seguidos de demora e dia de atendimento ( $16,1 \%$ e $9,7 \%$ respectivamente).

No que tange aos motivos do abandono relacionados à equipe profissional apenas uma pessoa $(1,6 \%)$ mencionou a insatisfação com o acompanhamento e outra $(1,6 \%)$ citou o relacionamento insatisfatório com profissional. Quanto aos motivos relacionados à própria pessoa, a maior parcela dos indivíduos respondeu que a razão que mais contribuiu para desistência do tratamento foi problemas no trabalho $(38,7 \%)$, seguida de falta de tempo (25,8\%). Motivos como, problemas familiares, dificuldade de transporte, problemas de saúde, mudança de cidade, problemas financeiros, não conseguir fazer a dieta e falta de motivação foram citados, porém em menor número. Apesar de algumas pessoas citarem mais de um motivo, o maior percentual foi de quem referiu somente um $(35,5 \%)$ e dois motivos $(33,9 \%)$ (Tabela 3$)$. 


\begin{tabular}{|c|c|c|}
\hline Variáveis & $\mathrm{n}$ & $\%$ \\
\hline \multicolumn{3}{|c|}{ Motivos relacionados à organização e estrutura do serviço } \\
\hline Horário de atendimento & 15 & 24,2 \\
\hline Dificuldade de agendar retorno & 15 & 24,2 \\
\hline Demora de atendimento & 10 & 16,1 \\
\hline Dia de atendimento & 6 & 9,7 \\
\hline Localização do serviço & 3 & 4,8 \\
\hline Dificuldade de realizar exames laboratório & 3 & 4,8 \\
\hline Disponibilidade de consulta com nutricionista & 1 & 1,6 \\
\hline \multicolumn{3}{|l|}{ Motivos relacionados à equipe profissional } \\
\hline Insatisfação com acompanhamento & 1 & 1,6 \\
\hline Relacionamento insatisfatório com profissional & 1 & 1,6 \\
\hline \multicolumn{3}{|l|}{ Motivos relacionados à pessoa } \\
\hline Problema no trabalho & 24 & 38,7 \\
\hline Falta de tempo & 16 & 25,8 \\
\hline Problemas familiares & 8 & 12,9 \\
\hline Dificuldade de transporte & 7 & 11,3 \\
\hline Problema de saúde & 6 & 9,7 \\
\hline Mudança de cidade & 4 & 6,5 \\
\hline Não conseguia fazer a dieta & 2 & 3,2 \\
\hline Problema financeiro & 2 & 3,2 \\
\hline Falta de motivação & 1 & 1,6 \\
\hline \multicolumn{3}{|l|}{ Número de motivos } \\
\hline Um motivo & 22 & 35,5 \\
\hline Dois motivos & 21 & 33,9 \\
\hline Três motivos & 12 & 19,4 \\
\hline Quatro motivos & 4 & 6,5 \\
\hline Cinco motivos & 2 & 3,2 \\
\hline Seis motivos & 1 & 1,6 \\
\hline
\end{tabular}

$\mathrm{Na}$ Tabela 4, encontram-se os dados referentes às condições de tratamento atual realizado pela população estudada depois da saída do PEPE. A maioria dos participantes $(87,1 \%)$, não está realizando nenhum tipo de tratamento para obesidade em outro serviço, e dos 8 indivíduos $(12,9 \%)$ que estão sendo acompanhados por outros serviços de saúde, 7 (87,5\%) informaram estão obtendo sucesso com o tratamento atual. Porém quando questionados sobre a perda de peso somente $32(51,6 \%)$ pessoas informaram que conseguiram. Quanto aos cuidados necessários para a perda e controle do peso, $54(87,1 \%)$ não estão fazendo dieta e 57 $(91,9 \%)$ não exercem nenhuma atividade física. 


\begin{tabular}{|c|c|c|}
\hline Variável & $\mathbf{n}$ & $\%$ \\
\hline \multicolumn{3}{|c|}{ Tratamento atual em outro serviço $(n=62)$} \\
\hline Não & 54 & 87,1 \\
\hline Sim & 8 & 12,9 \\
\hline \multicolumn{3}{|c|}{ Acha que teve sucesso no tratamento atual $(n=8)$} \\
\hline Não & 1 & 12,5 \\
\hline Sim & 7 & 87,5 \\
\hline \multicolumn{3}{|c|}{ Perdeu peso após sair do Pepe $(n=62)$} \\
\hline Não & 32 & 51,6 \\
\hline Sim & 25 & 40,3 \\
\hline Não sabe informar & 5 & 8,1 \\
\hline \multicolumn{3}{|c|}{ Atualmente faz dieta $(n=62)$} \\
\hline Não & 54 & 87,1 \\
\hline Sim & 8 & 12,9 \\
\hline \multicolumn{3}{|c|}{ Atualmente faz atividade física $(n=62)$} \\
\hline Não & 57 & 91,9 \\
\hline Sim & 5 & 8,1 \\
\hline
\end{tabular}

\section{Discussão}

No que concerne às variáveis sociodemográficas dos participantes, trata-se de uma população com características semelhantes a populações de outros estudos realizados com pessoas com excesso de peso, principalmente se tratando da faixa etária a partir dos 30 anos de idade ${ }^{14}$. O fato de a maioria ser majoritariamente do sexo feminino pode ser explicada por questões inerentes ao gênero, pois de acordo com estudos, as mulheres procuram utilizar mais os serviços de saúde para tratamento ambulatorial e para controle de obesidade ${ }^{15}$. Além das mulheres estarem mais preocupadas com fatores relacionados à saúde e a prevenção de doenças do que o sexo masculino, quando se trata de excesso de peso a insatisfação com a imagem corporal leva as mesmas a procurarem tratamento com o propósito não só de cuidado com a saúde, mas também preocupação com a aparência ${ }^{16,17}$.

Vale ressaltar que as características da população do estudo no que tange a baixa renda e escolaridade são importantes, pois são pessoas com maior vulnerabilidade e menor possibilidade de encontrar outros serviços de saúde para o acompanhamento e controle do seu problema de saúde, podendo assim desenvolver complicações inerentes ao excesso de peso. Determinantes sociais como escolaridade e renda tem uma dimensão maior no controle do peso quando se refere à continuidade de uma terapêutica, pois esta envolve mudanças de hábitos de vida que demanda das pessoas condições financeira para facilitar aquisição de novos hábitos saudáveis, tais como acesso a alimentos de maior qualidade, tempo e estrutura adequada para prática de atividade física ${ }^{18}$.

Geralmente, a obesidade vem acompanhada de outras comorbidades, como hipertensão arterial, diabetes mellitus e dislipidemias. Neste estudo não foi diferente, pois a frequência de pessoas com alterações com mais de uma comorbidade foi relevante. Ressalta-se que este perfil apresentado pela população desistente do tratamento tem importância à medida que se sabe que as complicações advindas da obesidade e comorbidades associadas podem se agravar se não forem adequadamente caracterizadas e acompanhadas. 
O presente estudo também evidenciou um alto percentual de pessoas que abandonaram o SUPRIMIDO em menos de um ano e realizou menos de cinco consultas. Pensa-se que o pouco tempo de frequência no programa não foi suficiente para criação de vínculo com a equipe e conhecer os benefícios do programa para atingir o objetivo principal esperado que seria, além do controle de peso, a melhora das condições clínicas e metabólicas em geral. Estes aspectos são essenciais na adesão ao tratamento, pois os pacientes estabelecem uma transferência positiva quando há um vínculo com o profissional, o que se torna benéfico à adesão ao tratamento ${ }^{18}$.

Os resultados mostram que o horário de atendimento está entre os principais motivos do abandono, semelhante, porém em menor proporção ao estudo de Leal ${ }^{19}$ que mostrou também horário inadequado como causa de desistência do tratamento da obesidade. A dificuldade de agendar retorno e a demora no atendimento foram motivos também encontrados e que são consonantes com os achados da pesquisa feita com crianças e adolescentes ${ }^{20}$. Acredita-se que esses motivos neste estudo estejam relacionados ao grande número de pacientes cadastrados no SUPRIMIDO, que não permite $o$ agendamento do retorno com mais brevidade. A sobrecarga na agenda do SUPRIMIDO, resulta em intervalos longos entre as consultas, o que pode desmotivar a permanência no programa.

Quanto à demora no atendimento, apesar de que na maioria das vezes ocorre porque o paciente é atendido por profissionais de diferentes especialidades (enfermagem, medicina, nutrição e psicologia) no mesmo dia, este aspecto deve ser mais bem explicado nos contatos inicias para que ele entenda o beneficio de cada atendimento e assim aceite melhor o tempo que permanece no serviço para que todos os atendimentos sejam realizados.

Apesar de algumas razões citadas relacionadas ao serviço, como o dia de atendimento, localização do serviço, dificuldade de realizar exames e indisponibilidade de consulta com algum profissional específico serem em menor número, merecem atenção, pois podem representar barreiras de acesso para a continuidade da terapêutica. Para a efetividade do atendimento e acompanhamento dos pacientes é necessário um ajuste na gestão do projeto para solucionar os motivos apresentados visando uma nova opção para continuidade do tratamento do maior número possível dos indivíduos participantes do programa.
O baixo percentual de insatisfação com o tratamento e com a equipe revela que estes não são fatores que desencadearam o abandono. Pensa-se assim, que os profissionais que participam do programa devem estar desempenhando suas atribuições de forma satisfatória, porém devem colaborar para a melhora da estrutura e organização do serviço, que representaram aspectos mais dificultadores da permanência do paciente. A existência de atenção, empatia e vínculos facilitam a cooperação mútua entre todos os envolvidos, pode representar em um dos principais elementos facilitadores da adesão ${ }^{11}$.

Quanto aos motivos relacionados à própria pessoa, os problemas no trabalho são os mais citados. Considerando que a população analisada pertencia em sua maioria a uma faixa etária ainda produtiva e que se ausentar do ambiente de trabalho nem sempre é uma condição facultativa, este pode ser um dos fatores já esperado. Os participantes que relacionaram a desistência de frequentar o programa com problemas relacionados ao trabalho referiram que o horário das consultas não era compatível com sua disponibilidade de tempo. Estes resultados estão de acordo com outro estudo, que constatou que o horário de trabalho pode ser um empecilho para a frequência às consultas em serviços de saúde ${ }^{20}$.

As dificuldades de transporte e problemas de saúde também aparecem como barreira para o seguimento no programa do presente estudo. Segundo relatos dos participantes a localização de sua residência não permite acesso fácil a transporte coletivo e sua condição financeira dificulta o seu deslocamento com transportes alternativos. Estudo prospectivo realizado com mulheres com excesso de peso apontou a falta de tempo, problemas pessoais e limitação de saúde como causa de abandono corroborando os achados desta pesquisa ${ }^{21}$.

Embora outra pesquisa aponte a falta de motivação e condições financeiras como causas de evasão em tratamento de obesidade ${ }^{20,23}$, neste estudo estes aspectos não representarem os motivos mais frequentes. Porém, os mesmos devem ser observados no acompanhamento de pessoas com obesidade, principalmente se tiveram baixa condição financeira, pois podem dificultar o acesso a determinados cuidados prescritos que são necessários para redução do peso ${ }^{11,21,23}$. É fundamental que os profissionais tenham conhecimento das condições socioeconómicas do paciente e que procure sempre estabelecer um 
diálogo que o estimule a expressar suas dificuldades, pois assim poderá encontrar estratégias para contorna-las, a exemplo da substituição de alimentos dispendiosos e difíceis de serem encontrados, por outros similares mais baratos e de fácil acesso ${ }^{11}$. Somente por meio de uma abordagem individualizada é possível auxiliar o paciente a superar as barreiras.

Assim, tanto os motivos observados neste estudo, quantos outros apontados pela literatura ${ }^{20,23}$, tais como, escolha de outros tratamentos, resultados insatisfatórios, dificuldades do enfrentamento da doença, os gastos com a mudança do estilo de vida, merecem ser considerados no acompanhamento do paciente a fim de colaborar com a não desistência da terapêutica.

A grande proporção de pessoas que após abandonar o PEPE, está sem nenhum acompanhamento, bem como sem perder peso, é uma situação preocupante, pois as mesmas podem vir a ter complicações relacionadas ao excesso de peso. A falta de uma terapêutica efetiva assistida por profissionais capacitados pode levá-las a realizar tratamentos inadequados comprometendo ainda mais a sua saúde.

\section{Conclusão}

O abandono do tratamento da obesidade é causado por múltiplos fatores que podem estar relacionados a motivos de ordem pessoal, à estrutura e organização do serviço de saúde a equipe de profissionais. Os motivos de ordem pessoal, como a falta de tempo e problemas inerentes ao trabalho foram os mais frequentes. Enquanto que os relacionados à estrutura e organização do serviço de saúde, o horário de atendimento e dificuldade em agendar retorno foram os motivos mais citados. Os relacionados à equipe, apesar de mencionados, foram por pequeno número dos participantes. Estes achados demandam uma reflexão cuidadosa e requer o envolvimento de todos envolvidos (pacientes, profissionais e gestores do serviço) no sentido de encontrar formas de amenizar as dificuldades.

Como limitações do estudo, destaca-se a presença de informações incompletas nos prontuários e o fato de não ter sido possível incluir todas as pessoas consideradas desistentes, o que pode reduzir o poder de generalização dos resultados. Porém, mesmo com apenas uma amostra da população, os resultados podem ser válidos para colaborar com o desenvolvimento de abordagens precoces no sentido de evi-tar o abandono. Como pesquisas relacionadas ao tema são escassas, o que dificulta a compreensão de uma abordagem mais ampliada junto aos pacientes de modo a contribuir com adesão e a permanência no programa, se faz necessários mais estudos sobre o mesmo.

\section{Contribuições das autoras}

Palmeira CS, Novais I e Santos VRS participaram da concepção e operacionalização do projeto, análise estatística e interpretação dos dados, redação do artigo e aprovação final da versão a ser publicada. Lima ML contribuiu na revisão crítica do conteúdo intelectual do artigo.

\section{Conflitos de interesses}

Nenhum conflito financeiro, legal ou político envolvendo terceiros (governo, empresas e fundações privadas, etc.) foi declarado para nenhum aspecto do trabalho submetido (incluindo mas não limitando-se a subvenções e financiamentos, participação em conselho consultivo, desenho de estudo, preparação de manuscrito, análise estatística, etc.).

\section{Referências}

1. Hruby A, Hu FB. The Epidemiology of Obesity: A Big Picture. Pharmaco economics. 2015;33(7):673-89. doi: 10.1007/s40273014-0243-x

2. Associação Brasileira para o Estudo da Obesidade e da Síndrome Metabólica. Diretrizes Brasileiras de Obesidade 2016 / ABESO - Associação Brasileira para o Estudo da Obesidade e da Síndrome Metabólica. 4.ed. São Paulo: 2016. p. 188.

3. Brasil. Ministério da Saúde. Secretaria de Vigilância em Saúde. Departamento de Vigilância de Doenças e Agravos não Transmissíveis e Promoção da Saúde. Vigitel Brasil 2017: vigilância de fatores de risco e proteção para doenças crônicas por inquérito telefônico: estimativas sobre frequência e distribuição sociodemográfica de fatores de risco e proteção para doenças crônicas nas capitais dos 26 estados brasileiros e no Distrito Federal em 2017. Brasília: Ministério da Saúde; 2018.

4. Serra-Majem L, Bautista-Castaño I. Etiology of obesity: two "key issues" and other emerging factors. Nutr Hosp. 2013;28 (Suppl 5):32-43. doi: 10.3305/nh.2013.28.sup5.6916 
5. Bhurosy T, Jeewon R. Overweight and obesity epidemic in developing countries: a problem with diet, physical activity, or socioeconomic status? Scientific World Journal. 2014:964236. doi: $\underline{10.1155 / 2014 / 964236}$

6. Macedo TTS, Palmeira CS, Guimarães AC, Lima ML, Ladeia AMT. The meaning of obesity: the perception of obese patients. Rev Enferm UFPE. 2013; 7(spe):7064-7073. doi: 10.5205/1981-8963-v7i 12a12377p7064-7073-2013

7. Sjöström L, Narbro K, Sjöström CD, Karason K, Wedel H, Lystig $T$ et al. Effects of bariatric surgery on mortality in Swedish obese subjects. N Engl J Med. 2007; 357(8):741-52. doi: 10.1056/ NEJMoa066254

8. Bueno JM, Leal FS, Saquy LPL, Santos CB, Ribeiro RPP. Educação alimentar na obesidade: adesão e resultados antropométricos. Rev Nutr. 2011; 24(4):575-584. doi: 10.1590/ $\underline{\mathrm{S} 1415-52732011000400006}$

9. Burgess E, Hassmén P, Welvaert M, Pumpa KL. Behavioural treatment strategies improve adherence to lifestyle intervention programmes in adults with obesity: a systematic review and metaanalysis. Clin Obes. 2017;7(2):105-14. doi: 10.1111/cob.12180

10. Koehnlein EA, Salado GA, Yamada AN. Adesão à reeducação alimentar para perda de peso: determinantes, resultados e a percepção do paciente. Rev Bras Nutr Clin. 2008; 23(1):56-65.

11. Palmeira CS, Garrido LMM, Santana P. Fatores intervenientes na adesão ao tratamento da obesidade. Cienc Enferm. 2016;22(1):11-22. doi: 10.4067/S0717-95532016000100002

12. Leão JM, Lisboa LCV, Pereira MA, Lima LF, Lacerda KC, Elias MRibeiro et al. Estágios motivacionais para mudança de comportamento em indivíduos que iniciam tratamento para perda de peso. J Bras Psiquiatr. 2015;64(2):107-14. doi: 10.1590/0047-2085000000065

13. World Health Organization. Obesity: preventing and managing the global epidemic. Report of a World Health Organization Consultation. Geneva: World Health Organization; 2000; p. 256.

14. Silva VS, Petroski EL, Souza I, Silva DAS. Prevalência e fatores associados ao excesso de peso em adultos do Brasil: um estudo de base populacional em todo território nacional. Rev Bras Ciênc Esporte. Florianópolis: 2012;34(3):713-726. doi: 10.1590/S010132892012000300013

15. Botton A, Cúnico SD, Strey MN. Diferenças de gênero no acesso aos serviços de saúde: problematizações necessárias. Mudanças - Psicologia da Saúde. 2017. 25(1): 67-72. doi: 10.15603/2176-1019/mud.v25n1p67-72
16. Araújo FM, González AD, Silva LC, Garanhani ML. Obesidade: possibilidades de existir e práticas de cuidado. Saúde soc. 2019; 28(2): 249-60. doi: 10.1590/S0104-12902019170152

17. Jones-Smith JC, Gordon-Larsen P, Siddiqi A, Popkin BM. Is the burden of overweight shifting to the poor across the globe? Time trends among women in 39 low- and middle-income countries (1991-2008). International Journal of Obesity; 2012; 36:1114-20. doi: 10.1038/ijo.2011.179

18. Trinca RT, Okuda LS. Vínculo profissional-paciente. Nutrição em Psiquiatria. $1^{\text {a }}$ ed. Porto Alegre: Editora Artmed; 2010; 3: 49-58.

19. Leal FS. Tratamento da obesidade: investigando o abandono e seus aspectos motivacionais [TESE]. Ribeirão Preto: Universidade de São Paulo; 2012.

20. Nogueira TFD, Zambon MP. Razões do abandono do tratamento de obesidade por crianças e adolescentes. Rev Paulista Pediátrica. São Paulo; 2013;31(3):338-43. doi: 10.1590/ S0103-05822013000300010

21. Soares AH, Oliveira C, Rocha TR, Cordoba GMC, Nobre JAS. Porque obesos abandonam o planejamento nutricional em uma clínica-escola de nutrição?. Revista Brasileira de Obesidade, Nutrição e Emagrecimento. 2017;11(66):368-75.

22. Amorim DCA, Coutinho CM, Palmeira CS. Treatment adherence of people with overweight. Rev Enferm UFPE on line. 2016;10(6):4788-96. doi: 10.5205/reuol.8200-71830-3SM.1006sup201610

23. Burgess $E$, Hassmén P, Pumpa KL. Determinants of adherence to lifestyle intervention in adults with obesity: a systematic review. Clin Obes. 2017;7(3):123-135. doi: 10.1111/cob.12183 